\title{
HIV-1 Transmitted Drug Resistance Mutations in Newly Diagnosed Antiretroviral-Naive Patients in Turkey
}

\author{
Murat Sayan, ${ }^{1,2}$ Fatma Sargin, ${ }^{3}$ Dilara Inan, ${ }^{4}$ Dilek Y. Sevgi, ${ }^{5}$ Aysel K. Celikbas, \\ Kadriye Yasar, ${ }^{7}$ Figen Kaptan, ${ }^{8}$ Selda Kutlu, ${ }^{9}$ Nuriye T. Fisgin, ${ }^{10}$ Ayse Inci, ${ }^{11}$ Nurgul Ceran, ${ }^{12}$ \\ Ilkay Karaoglan, ${ }^{13}$ Atahan Cagatay, ${ }^{14}$ Mustafa K. Celen, ${ }^{15}$ Suda T. Koruk, ${ }^{16}$ Bahadir Ceylan, ${ }^{17}$ \\ Taner Yildirmak, ${ }^{18}$ Halis Akalın, ${ }^{19}$ Volkan Korten, ${ }^{20}$ and Ayse Willke ${ }^{21}$
}

\begin{abstract}
HIV-1 replication is rapid and highly error-prone. Transmission of a drug-resistant HIV-1 strain is possible and occurs within the HIV-1-infected population. In this study, we aimed to determine the prevalence of transmitted drug resistance mutations (TDRMs) in 1,306 newly diagnosed untreated HIV-1-infected patients from 21 cities across six regions of Turkey between 2010 and 2015. TDRMs were identified according to the criteria provided by the World Health Organization's 2009 list of surveillance drug resistance mutations. The HIV-1 TDRM prevalence was $10.1 \%(133 / 1,306)$ in Turkey. Primary drug resistance mutations (K65R, M184V) and thymidine analogue-associated mutations (TAMs) were evaluated together as nucleos(t)ide reverse transcriptase inhibitor (NRTI) mutations. NRTI TDRMs were found in $8.1 \%(107 / 1,306)$ of patients. However, TAMs were divided into three categories and M41L, L210W, and T215Y mutations were found for TAM1 in $97(7.4 \%)$ patients, D67N, $\mathrm{K} 70 \mathrm{R}, \mathrm{K} 219 \mathrm{E} / \mathrm{Q} / \mathrm{N} / \mathrm{R}, \mathrm{T} 215 \mathrm{~F}$, and T215C/D/S mutations were detected for TAM2 in $52(3.9 \%)$ patients, and $\mathrm{M} 41 \mathrm{~L}+\mathrm{K} 219 \mathrm{~N}$ and M41L + T215C/D/S mutations were detected for the TAM1 + TAM2 profile in $22(1.7 \%)$ patients, respectively. Nonnucleoside reverse transcriptase inhibitor-associated TDRMs were detected in $3.3 \%$ (44/1,306) of patients (L100I, K101E/P, K103N/S, V179F, Y188H/L/M, Y181I/C, and G190A/E/S) and TDRMs to protease inhibitors were detected in $2.3 \%(30 / 1,306)$ of patients $(\mathrm{M} 46 \mathrm{~L}, \mathrm{I} 50 \mathrm{~V}, \mathrm{I} 54 \mathrm{~V}$, Q58E, L76V, $\mathrm{V} 82 \mathrm{~A} / \mathrm{C} / \mathrm{L} / \mathrm{T}, \mathrm{N} 83 \mathrm{D}, \mathrm{I} 84 \mathrm{~V}$, and L90M). In conclusion, long-term and large-scale monitoring of regional levels of HIV-1 TDRMs informs treatment guidelines and provides feedback on the success of HIV-1 prevention and treatment efforts.
\end{abstract}

\footnotetext{
${ }^{1}$ Faculty of Medicine, Clinical Laboratory, PCR Unit, University of Kocaeli, Kocaeli, Turkey.

${ }^{2}$ Research Center of Experimental Health Sciences, University of Near East, Nicosia, Northern Cyprus.

${ }^{3}$ Goztepe Educational and Research Hospital, Clinic of Infectious Diseases, Medeniyet University, Istanbul, Turkey.

${ }^{4}$ Faculty of Medicine, Department of Infectious Diseases, University of Akdeniz, Antalya, Turkey.

${ }^{5}$ Educational and Research Hospital, Clinic of Infectious Diseases, Sisli Etfal, Istanbul, Turkey.

${ }^{6}$ Educational and Research Hospital, Clinic of Infectious Diseases, Ankara Numune, Ankara, Turkey.

${ }^{7}$ Educational and Research Hospital, Clinic of Infectious Diseases, Bakırkoy Dr. Sadi Konuk, Istanbul,Turkey.

${ }_{9}^{8}$ Educational and Research Hospital, Department of Infectious Diseases, University of Katip Celebi, İzmir, Turkey.

${ }^{9}$ Faculty of Medicine, Department of Infectious Diseases, University of Pamukkale, Denizli, Turkey.

${ }^{10}$ Faculty of Medicine, Department of Infectious Diseases, University of 19 May1s, Samsun, Turkey.

${ }^{11}$ Educational and Research Hospital, Clinic of Infectious Diseases, Istanbul Kanuni Sultan Süleyman, Istanbul, Turkey.

${ }^{12}$ Educational and Research Hospital, Clinic of Infectious Diseases, Haydarpasa Numune, Istanbul, Turkey.

${ }^{13}$ Faculty of Medicine, Department of Infectious Diseases, University of Gaziantep, Gaziantep, Turkey.

${ }^{14}$ Faculty of Medicine, Department of Infectious Diseases, University of Istanbul, Istanbul, Turkey.

${ }^{15}$ Faculty of Medicine, Department of Infectious Diseases, University of Dicle, Diyarbakır, Turkey.

${ }^{16}$ Faculty of Medicine, Department of Infectious Diseases, University of Harran, Urfa, Turkey.

${ }^{17}$ Faculty of Medicine, Department of Infectious Diseases, University of Bezm-i Alem, Istanbul, Turkey.

${ }^{18}$ Educational and Research Hospital, Clinic of Infectious Diseases, Istanbul Okmeydani, Istanbul, Turkey.

${ }^{19}$ Faculty of Medicine, Department of Infectious Diseases, University of Uludag, Bursa, Turkey.

${ }^{20}$ Faculty of Medicine, Department of Infectious Diseases, University of Marmara, Istanbul, Turkey.

${ }^{21}$ Faculty of Medicine, Department of Infectious Diseases, University of Kocaeli, Kocaeli, Turkey.
} 


\section{Introduction}

$\mathbf{T}$ ODAY, TREATMENT OF HIV-1 INFECTION is based on a combination of three or more targeted drugs and is referred to as highly active antiretroviral therapy (HAART). A combination of two nucleoside/nucleotide reverse transcriptase inhibitors (NRTIs) and a third agent, which may be selected from nonnucleoside reverse transcriptase inhibitors (NNRTIs), one of several ritonavir-boosted protease inhibitors (PIs), or the new class of integrase strand transfer inhibitors (INSTIs), is currently recommended for first-line therapy. ${ }^{1}$

A major cause of antiretroviral resistance mutations in newly diagnosed HIV-1-infected patient is transmission of this strain from another HIV-1-infected individual. ${ }^{2}$ The turnover of the HIV-1 population is rapid (approximately 1 day) and error-prone (mutation rate ca. $3 \times 10^{-5}$ mutations/base/replication cycle), resulting in a large and genetically diverse population in vivo in which resistance may emerge. ${ }^{3}$ Analysis of the kinetics of emergence of drug resistance in vivo suggests that many single nucleotide mutations conferring drug resistance may be present prior to the start of HAART. ${ }^{4}$ In 2004, the European HIV Drug Resistance Guidelines Panel presented recommendations for the use of initial HIV-1 drug resistance testing managing treatment for HIV-1 infection. ${ }^{5}$ However, all current guidelines recommend HIV-1 drug resistance testing for all HIV-1-infected patients prior to therapy initiation. ${ }^{1,6,7}$

The World Health Organization (WHO) is conducting a global surveillance of transmitted HIV-1 drug resistance. Transmitted HIV-1 drug resistance is classified into three categories according to this surveillance: low prevalence $(<5 \%)$, moderate prevalence $(5-15 \%)$, and high prevalence $(>15 \%) .{ }^{8}$ In a population, genotypic resistance testing is considered cost effective for HIV-1 infection when the level of transmitted drug resistance is $>5 \%$. $^{9}$

According to the official HIV/AIDS annual surveillance data of the Turkey Ministry of Health, 1,767 patients were newly diagnosed with HIV-1 in 2014. In the period between 1985 and 2014 there were only 9,379 cumulative HIV/AIDS cases in Turkey, and so by the end of 2014, the cumulative increase in HIV-1 patients was $38 \% .{ }^{10}$ According to the IMS Health Turkey there are 4,117 HIV-1-infected patients under antiretroviral therapy (ART). ${ }^{11}$ However, there is limited knowledge of transmitted drug resistance mutations (TDRMs) of HIV-1 strains in Turkish patients. In a single study with 117 newly diagnosed HIV-1-infected Turkish cases, the prevalence of TDRMs was $7.6 \% .^{12}$

The objective of this study is to accurately determine and to understand the circulation of TDRMs of HIV-1 in newly diagnosed, untreated patients from a cohort consisting of individuals from cities in all regions of Turkey.

\section{Materials and Methods}

\section{Patient population}

The present study was conducted between March 2010 and March 2015, and it included 1,306 HIV-1-infected patients who were newly diagnosed in infectious disease departments of 21 cities from all regions of Turkey. The clinic and laboratory characteristics of the patients are shown in Table 1. The study was approved by the local ethics committee (Clinical Research Ethics Committee of Kocaeli University), and written informed consent was obtained from each patient. All of the patients were categorized as HIV carriers according to European AIDS Clinical Society (EACS) Guidelines. ${ }^{1}$ Based on records of the Turkey Ministry of Health, the study patients were newly diagnosed and were ART-naive. The U.S. Centers for Disease Control and Prevention (CDC) classification system was used to determine the HIV infection staging of patients. ${ }^{13}$

Blood samples with K2EDTA were separated by centrifugation immediately, aliquoted, and then kept at $-80^{\circ} \mathrm{C}$ until testing. Anti-HIV-1/2 antibody was tested using commercially available microparticle enzyme immunoassay kits (Axsym; Abbott Laboratories, Abbott Park, IL, and Elecsys, Roche Diagnostics, Mannheim, Germany). All of the samples that were anti-HIV positive by ELISA at least two times were confirmed by a Western blot test (DIA PRO, HIV-1 LIA, Diagnostic Bioprobes Srl, Milano, Italy) in the Istanbul Venereal Diseases Hospital in Turkey. To maintain subject confidentiality, a unique identification number was assigned to each specimen.

\section{HIV-1 RNA detection}

HIV-1 RNA was detected and quantified by a commercial real-time PCR assay-QIAsypmhony + Rotorgene Q/artus HIV-1 QS-RGQ v1 (Qiagen GmBH, Hilden, Germany), COBAS Ampliprep/COBAS TaqMan HIV-1 Test (Roche Molecular Systems, Inc., Pleasanton, CA), and Abbott M2000 SP/ Abbott RealTime HIV-1 Amplification Kit (Abbott Molecular Inc., Des Plaines, IL).

\section{PCR amplification and sequence analysis}

A genotypic resistance test was performed by population sequencing of the viral protease and part of the reverse transcriptase (RT) using an in-house method. Specific primer pairs were designed according to the ANRS (AIDS National Research Agency) drug resistance interpretation algorithm (www.hivfrenchresistance.org). The polymerase chain reaction (PCR) condition was applied as following: RT (codons 41238): outer primers (798 bp); MJ3: 5'-agtaggacctacacctgtca-3' $(2,480$ to 2,499$)$ and MJ4: $5^{\prime}$-ctgttagtgctttggttcctct-3' $(3,399$ to $3,420)$, inner primers (573 bp) A(35): $5^{\prime}$-ttggttgcactttaaatttt cccattagtcctatt- $3^{\prime}\left(2,530\right.$ to 2,558) and NE1(35): $5^{\prime}$-cctactaactt ctgtatgtcattgacagtccagct- $3^{\prime} \quad(3,300$ to 3,334$)$. Sequencing primer; $A(20)$ : $5^{\prime}$-attttcccattagtcctatt- $3^{\prime}$. Protease (codons 2390): outer primers: $5^{\prime}$ prot $1: 5^{\prime}$ - taatttttagggaagatctggccttcc- $3^{\prime}$ $(2,082$ to 2,109$)$ and $3^{\prime}$ prot 1 : $5^{\prime}$-gcaaatactggagtattgtatggatttt cagg-3' (2,703 to 2,734), inner (amplification: 507-bp fragment) and sequencing primers $5^{\prime}$ prot 2: $5^{\prime}$-tcagagcagaccaga gccaacagccca-3' $(2,136$ to 2,163$)$ and $3^{\prime}$ prot 2: $5^{\prime}$-aatgett ttatttttcttctgtcaatggc-3' $(2,621$ to 2,650$)$. HIV-1 cDNA synthesis was done with the First Strand cDNA Synthesis Kit (Thermo Scientific Inc., Fermentas, Lithuania) including the $\mathrm{M}-\mathrm{MuLV}$ reverse transcriptase enzyme. The PCR conditions were $95^{\circ} \mathrm{C}$ for $10 \mathrm{~min}$, and then 45 cycles consisting of $95^{\circ} \mathrm{C}$ for $45 \mathrm{~s}, 55^{\circ} \mathrm{C}$ for $45 \mathrm{~s}$, and $72^{\circ} \mathrm{C}$ for $45 \mathrm{~s}^{12}$

All PCR products were purified using the Highly Pure PCR Product Purification Kit (Roche Diagnostics GmbH, Mannheim, Germany) and directly sequenced with ABI PRISM 310 Genetic Analyzer equipment using the DYEnamic ET Terminator Cycle Sequencing Kit (Amersham Pharmacia Biotech Inc., Piscataway, NJ). The following thermal protocol 
Table 1. Demographic Characteristics of the Patients Infected with HIV-1

\begin{tabular}{|c|c|c|c|c|}
\hline Characteristic & \multicolumn{4}{|c|}{ Study group } \\
\hline $\begin{array}{l}\text { Patient, } n \\
\text { Gender, M/F }(\%) \\
\text { Age, median years (range) } \\
\text { CD4 }{ }^{+} \text {T-cell count, median } \mathrm{mm}^{3} \text { (range) } \\
\text { HIV-RNA load, median IU/ml (range) }\end{array}$ & & $\begin{array}{r}1151 \\
361 \\
2.59+\mathrm{E} 6(6\end{array}$ & $\begin{array}{l}88 / 12) \\
4) \\
351) \\
2-3.29+E 6)\end{array}$ & \\
\hline HIV-1 subtype, $n(\%)$ & $\begin{array}{l}\text { Subtype B } \\
\text { Non-subtype B }\end{array}$ & $\begin{array}{l}885(68) \\
136(10)\end{array}$ & $\begin{array}{l}\text { A1 } \\
\text { C } \\
\text { D } \\
\text { F } \\
\text { F1 } \\
\text { F2 } \\
\text { G } \\
\text { K }\end{array}$ & $\begin{array}{r}- \\
48(3.6) \\
21(1.6) \\
3(0.2) \\
2(0.1) \\
24(1.8) \\
1(0.07 \\
36(2.7) \\
1(0.07\end{array}$ \\
\hline & $\begin{array}{l}\text { Circulating } \\
\text { recombinant } \\
\text { form }(\mathrm{CRF})\end{array}$ & $285(22)$ & $\begin{array}{l}\text { CRF01_AE } \\
\text { CRF 02_AG } \\
\text { CRF 03_AB } \\
\text { CRF 06_cpx } \\
\text { CRF 07_BC } \\
\text { CRF 08_BC } \\
\text { CRF 11_cpx } \\
\text { CRF 12_BF } \\
\text { CRF 13_cpx } \\
\text { CRF 14_BG }\end{array}$ & $\begin{aligned} 132 & (10.1 \\
85 & (6.5) \\
13 & (1) \\
3 & (0.2) \\
1 & (0.07 \\
1 & (0.07 \\
3 & (0.2) \\
33 & (2.5) \\
3 & (0.2) \\
11 & (0.8)\end{aligned}$ \\
\hline
\end{tabular}

Sampling, region/city of Turkey

Acquisition route, $n(\%)$

Coinfection status, $n(\%)$
Marmara/Kocaeli, İstanbul, Edirne, Bursa, Sakarya

Black Sea/Samsun, Artvin, Giresun, Trabzon, Bolu

Southeast Anatolia/Urfa, Diyarbakir, Gaziantep

Central Anatolia/Ankara, Kayseri

Aegean/Izmir, Denizli, Çanakkale

Mediterranean/Antalya, Adana, Mersin

Heterosexual contact

MSM

Bisexual contact

Blood transfusion

Injection drug use

Tattoo

Dental/medical surgery

Breast-feeding

Vertical route

Total

Hepatitis B

Syphilis

Tuberculosis

$P$. jiroveci pneumonia

Hepatitis C

Kaposi sarcoma

Candida esophagitis

HPV infection

Hepatitis D

Herpes zoster

Toxoplasmosis

Condyloma

CMV retinitis

Cryptococcal meningitis

PML

Total
$674(52)$

$563(43)$

$47(3.6)$

$8(0.6)$

$4(0.3)$

$4(0.3)$

$2(0.1)$

$2(0.1)$

$2(0.1)$

1,306 (100)

35 (2.7)

$18(1.4)$

$14(1.1)$

$11(0.8)$

$7(0.5)$

$7(0.5)$

$7(0.5)$

$4(0.3)$

$3(0.2)$

$3(0.2)$

$3(0.2)$

$2(0.15)$

$1(<0.1)$

$1(<0.1)$

$1(<0.1)$

$117(8.9)$

M/F, male/female; MSM, men who have sex with men; HPV, human papilloma virus; CMV, cytomegaloviru; PML, progressive multifocal leukoencephalopathy. 
was used for the cycle sequencing: 35 cycles consisting of $95^{\circ} \mathrm{C}$ for $20 \mathrm{~s}, 50^{\circ} \mathrm{C}$ for $25 \mathrm{~s}$, and finally $60^{\circ} \mathrm{C}$ for $2 \mathrm{~min}$. The sequences obtained with an electropherogram were assembled using Vector NTI v.5.1 (InforMax, Invitrogen, Life Science Software, Frederick, MD).

\section{Drug resistance mutation detection}

Drug resistance mutations were analyzed using the Stanford HIV Drug Resistance Database (www.hivdb.stanford.edu), and TDRMs were defined according to the mutation list published for the surveillance of TDRMs (SDRM) as recommended by the WHO. The WHO SDRM list included only consensus nonpolymorphic drug resistance mutations at 43 positions in HIV-1 protease and RT. Selected mutations were defined as those occurring at a prevalence $\leq 0.5 \%$ in ARTnaive individuals in subtypes for which $>1,000$ sequences were available. ${ }^{14}$ However, thymidine analogue-associated mutations (TAMs) were evaluated for the first time in Turkish patients in two distinct genotypic profiles: TAM1 and TAM2, as well as in TAM1 + TAM2 profiles.

\section{HIV-1 subtyping}

The HIV-1 subtype was determined by use of the HIVdb Stanford University and geno2pheno (http://coreceptor.bioinf .mpi-inf.mpg.de) subtyping tools. The information was then compared to the consensus subtype B reference sequence, and the differences were used as query parameters to interrogate the HIV database as rapid computer-assisted virtual phenotyping.

The submitted nucleotide sequences were assigned GenBank accession numbers KT284379-KT285684.

\section{Statistical analysis}

Differences between two proportions were measured using Pearson's $\chi^{2}$ test or Fisher's exact test (see Supplementary data: Fisher Exact Test; Supplementary Data are available online at www.liebertpub.com/aid). $p \leq 0.05$ was considered statistically significant. Statistical analyses were performed using SPSS for Windows statistical software (SPSS Inc., Chicago, IL).

\section{Results}

The average TDRM prevalence was detected as $10.1 \%$ (133/ 1306) in newly diagnosed HIV-1-infected patients in Turkey.
The frequencies of the primary drug resistance for NRTIs, NNRTIs, and PIs were $0.6 \%$ (8/1,306), 3.3\% (44/1,306), and $2.3 \%$ (30/1,306), respectively. However, most of the NRTI TDRMs were TAMs and were evaluated in three categories as TAM1, TAM2, and TAM1 + TAM2 in the NRTI class (Table 2). The frequency of the TDRM for NRTI + TAMs was $8.1 \%$ (107/1,306). K65R and M184V for the NRTI class, L100I, K101E, K101P, K103N, K103S, V179F, Y188H, Y188L, Y188M, Y181I, Y181C, G190A, G190E, and G190S for the NNRTI class, and M46L, I50V, I54V, Q58E, L76V, V82A, V82C, V82L, V82T, N83D, I84V, and L90M for the PI class were found as primary drug resistance mutations. Some HIV-1 TDRMs were discovered for the first time in Turkish patientsthese are L100I, V179F, and Y188M for the NNRTI and I50V, Q58E, and V82A/C for the PI drug class. However, the differences between NRTI, NNRTI, and PI drug class resistance prevalence were not significant (Fisher's exact test, $p \geq 0.05$ ). In addition, the TDRM prevalences between the present data and our recently unpublished (in 2014, $n=774$ and sampling from newly diagnosed, treatment-naive HIV-1-infected Turkish patients) data were not significant (Fisher's exact test, $p \geq 0.05$ ).

The prevalences of the TAM1, TAM2, and TAM1 + TAM2 profiles and the particular mutations present were as follows: 7.4\% (97/1306) and M41L, L210W, and T215Y for TAM1, $3.9 \%(52 / 1306)$ and D67N, K70R, K219E/Q/N/R, T215F, and $\mathrm{T} 215 \mathrm{C} / \mathrm{D} / \mathrm{S}$ for TAM2, and $0.7 \%$ (10/1306) and M41L + $\mathrm{K} 219 \mathrm{~N}$ and M41L + T215C/D/S for TAM1 + TAM2 (Table 2). Differences in the prevalence of TAM1 vs. TAM2, TAM1 vs. TAM1 + TAM2, and TAM2 vs. TAM1 + TAM2 were significant (Fisher's exact test, $p=0.01, p=0.02$, and $p=0.02$, respectively).

HIV-1 subtyping results are shown in three categories. According to the results, subtype B was dominant, found in $68 \%$ $(885 / 1,306)$ of study patients, while non-subtype B and circulating recombinant form (CRF) subtypes were found in $10 \%$ $(136 / 1,306)$ and $22 \%(285 / 1,306)$, respectively. The difference in the prevalence of HIV-1 subtypes was not significant (Fisher's exact test, $p \geq 0.05$ ). The non-subtype B category included five subtypes (C, D, F, G, and $\mathrm{K}$ ) and three subsubtypes (A1, F1, and F2). However, seven CRF subtypes (CRF01_AE, CRF02_AG, CRF03_AB, CRF07_BC, CRF08_BC, CRF12_BF, and CRF14_BG) and three cpx subtypes (CRF06_cpx, CRF11_cpx, and CRF13_cpx) were found (Table 1).

Table 2. Primary Drug Resistance Mutations in Newly Diagnosed HiV-1-Infected Patients in TuRkey (Between 2010 and 2015, $\mathrm{N}=1,306$ )

\begin{tabular}{llrr}
\hline Drug class & \multicolumn{1}{c}{${\text { Drug resistance } \text { mutation }^{\mathrm{a}}}^{\mathrm{c}}$} & $\mathrm{n}^{\mathrm{b}}$ & $\%$ \\
\hline NRTI & K65R, M184V & 8 & 0.6 \\
TAM1 & M41L, L210W, T215Y & 97 & 7.4 \\
TAM2 & D67N, K70R, K219E/Q/N/R, T215F, T215C/D/S & 52 & 3.9 \\
TAM1 + TAM2 & M41L + K219N, M41L + T215C/D/S & 107 & 0.7 \\
& & 44 & 3.3 \\
NNRTI & L100I, K101E/P, K103N/S, V179F, Y188H/L/M, Y181I/C, G190A/E/S & 44 & 2.3 \\
PI & M46L, I50V, I54V, Q58E, L76V, V82A/C/L/T, N83D, I84V, L90M & 30 & 10.1 \\
$\quad$ Total & & 133 & \\
\hline
\end{tabular}

\footnotetext{
${ }^{\mathrm{a}}$ Subtype of HIV-1 is not a variable in the identification of drug resistance mutation.

${ }^{\mathrm{b}}$ The number of $n$ consisted of each mutation detected patient.

NRTI, nucleoside reverse transcriptase inhibitors; NNRTI, nonnucleoside reverse transcriptase inhibitors; PI, protease inhibitors; TAM, thymidine analogue-associated mutation.
} 


\section{Discussion}

In the past few years, HIV-1 TDRMs have been analyzed all around the world. Previous studies have found the risk of transmitted HIV-1 drug resistance to be of $5-18 \%$ in the United States and Europe, $13.8 \%$ in Asia, and $2.2-24 \%$ in Africa. ${ }^{15-18}$ According to our unpublished study in 2014, the prevalence of HIV-1 TDRMs was found to be $6.7 \%$ in 774 newly diagnosed treatment-naive Turkish patients. ${ }^{19}$ Although new TDRMs of HIV-1 were found here, the prevalence was higher than our previously published and/or unpublished reports. However, the prevalence of HIV-1 TDRMs is still moderate in Turkey. This moderate assessment of TDRM frequency may influence the costs necessary for the analysis and management of ART-naive Turkish patients. The low median $\mathrm{CD}^{+} \mathrm{T}$ cell count in the study suggested that many of the patients may be late presenters. This may affect the level and TDRM type in Turkey. However, our results still show that genotypic resistance testing must remain an integral part of the management of HIV-1 infection in Turkey.

There are some similarities in surveillance strategies between our study and The SPREAD Programme of Europe: both studies used a population sequencing technique, accumulated surveillance data for the long term, sampled randomly from multiple areas, and had a large patient size for newly diagnosed HIV-1 infection. The average prevalence of TDRM in the SPREAD Programme was $8.4 \%$ and the frequencies of NRTI, NNRTI, and PI resistance were $4.7 \%, 2.3 \%$, and $2.9 \%$, respectively. ${ }^{20}$ Turkey showed a higher average prevalence than the SPREAD Programme. This higher TDRM prevalence and the discrepancy in NRTI resistance between Turkey and Europe may be associated with TAMs. ${ }^{18}$ Furthermore, the surveillance periods in both studies are different. Our study was performed in 2010-2015, but the SPREAD programme was performed in 2002-2006. However, the backbone treatment using mostly tenofovir + emtricitabine ( $~ 81 \%$, unpublished data) in Turkey and the lower circulation or decreasing K65R and M184V mutation frequency are important for firstline therapy in HIV-1-infected patients. The lower prevalence of NRTI backbone treatment mutations and the higher prevalence of TAMs may be associated with an increase in HIV-1 infection in men who have sex with men (MSM) in the past 2 years (in 2012, $23 \%$ of patients were MSM, compared to $43 \%$ in this study). ${ }^{12}$

Significant proportions of the patients with TDRMs have revertant TAMs, which transmit but do not reduce drug susceptibility. The largest proportion of TAMs included first by position of $215 \mathrm{C} / \mathrm{D} / \mathrm{F} / \mathrm{S} / \mathrm{Y}$ and second D67N, K219Q, or M41L mutations. ${ }^{21-23}$ TAMs are shown in Table 2, but have been evaluated separately in TAM1, TAM2, and TAM1 + TAM2 genotypic profiles. Our analysis clearly shows that TAM1 profile mutations are significantly more prevalent than TAM 2 or TAM $1+$ TAM 2 in Turkey $(p \geq 0.05)$. This may be due to M41L, which frequently occurred as a single resistance mutation in untreated patients.

However, according to new findings, the detection of a single M41L mutation at baseline did not influence the development of resistance in vitro or virological outcome on a tenofovir-containing regimen in patients. ${ }^{24}$ In addition, codon 215 is known to be atypical or a partial revertant amino acid position, which occurs in approximately $3 \%$ of newly diagnosed patients in the United States. ${ }^{25,26}$ In Turkey, the T215F and $\mathrm{T} 215 \mathrm{C} / \mathrm{D} / \mathrm{S}$ mutations were categorized in TAM 2 and in our study the proportion of TAM2 was determined to be $3.9 \%$ (Table 2). However, there are no previous data available on the frequency and type of TAM1 and TAM2 profiles in HIV-1infected patients in Turkey, and this study provides these data for the first time.

The molecular evidence from the present study indicates that subtype $\mathrm{B}$ is the most prevalent subtype among newly diagnosed HIV-1-infected patients in Turkey. However, trends in HIV-1 circulation in Turkey seem substantially heterogeneous with five non-subtype $B$, three non-subsubtype B, seven CRF subtypes, and three cpx subtypes of HIV-1 in spite of the dominance of subtype B. This heterogeneity may be associated with demographic changes in Turkey due to its specific geographic location: an increase in the number of refugees, especially from Syria (nearly 1.7 million since the 2011 crisis) and several countries from Africa, an increase in asylum seekers (nearly 100,000 as of January 2015, originating mainly from Iraq, Afghanistan, the Islamic Republic of Iran, and Somalia), ongoing human trafficking ( $84 \%$ suspected to be for sexual exploitation), and movements of tourists. ${ }^{27-29}$ Subtype diversity of HIV-1 is a major challenge in the development of a global control strategy for HIV-1 circulation.

In conclusion, moderate HIV-1 TDRM prevalence shows that drug resistance testing must remain an integral part of the management of newly diagnosed HIV-1-infected patients in Turkey. In addition, long-term and large-scale monitoring on regional levels of HIV-1 TDRMs informs treatment guidelines and provides feedback on the success of HIV-1 prevention and treatment efforts.

\section{Acknowledgments}

We thank Dr. Alison Lynn Hill (from Harvard University, Cambridge, MA) for English language editing. Ethical approval was provided by KOU KAEK 201345.

\section{Author Disclosure Statement}

No competing financial interests exist.

\section{References}

1. European AIDS Clinical Society (EACS) Guidelines. (2013): Version 7.0, October 2013. www.europeanaidsclinicalsociety .org.

2. Zolopa AR: The evolution of HIV treatment guidelines: Current state-of-the art of ART. Antiviral Res 2010;85:241244.

3. Perelson AS, Neumann AU, Markowitz M, et al.: HIV-1 dynamics in vivo: Virion clearance rate, infected cell life-span, and viral generation time. Science 1996;271:1582-1586.

4. Cortez KJ and Maldarelli F: Clinical management of HIV drug resistance. Viruses 2011;3:347-378.

5. Vandamme AM, Camacho RJ, Silberstein FC, et al.: European recommendations for the clinical use of HIV drug resistance testing: 2011 Update. AIDS Rev 2011;13:77-108.

6. British HIV Association guidelines for the treatment of HIV1-positive adults with antiretroviral therapy 2012 (Updated November 2013). HIV Med 2014;15(Suppl. 1):1-85.

7. Department of Health and Human Services: Guidelines for the use of antiretroviral agents in HIV-1-infected adults and adolescents. November 13, 2014. Available at www.aidsinfo nih.gov. 
8. Bennett DE, Myatt M, Bertagnolio S, et al.: Recommendations for surveillance of transmitted HIV drug resistance in countries scaling up antiretroviral treatment. Antiviral Ther 2008;13(Suppl. 2):25-36.

9. Sax P, Islam R, Walensky R, et al.: Should resistance testing be performed for treatment-naive HIV-infected patients? A costeffectiveness analysis. Clin Infect Dis 2005;41:1316-1323.

10. Hacettepe University, HIV/AIDS Treatment and Research Centre. The 2014 Annual Report. www.hatam.hacettepe.edu.tr.

11. IMS Health Turkey, August 2015: www.imshealth.com/ portal/site/imshealth.

12. Sayan M, Willke A, Ozgunes N, et al.: HIV-1 subtypes and primary antiretroviral resistance mutations in antiretroviral therapy naive HIV-1 infected individuals in Turkey. Jpn J Infect Dis 2013;66:306-311.

13. Centers for Disease Control and Prevention: Guidelines for national human immunodeficiency virus case surveillance, including monitoring for human immunodeficiency virus infection and acquired immunodeficiency syndrome. MMWR Recomm Rep Dec 1999;10;48:1-27.

14. Bennett DE, Camacho RJ, Otelea D, et al.: Drug resistance mutations for surveillance of transmitted HIV-1 drugresistance: 2009 update. PLoS One 2009;4:e4724.

15. Alteri C, Svicher V, Gori C, et al.: Characterization of the patterns of drug-resistance mutations in newly diagnosed HIV-1 infected patients naive to the antiretroviral drugs. BMC Infect Dis 2009;9:1-11.

16. Vercauteren J, Derdelinckx I, Sasse A, et al.: Prevalence and epidemiology of HIV type 1 drug resistance among newly diagnosed therapy-naive patients in Belgium from 2003 to 2006. AIDS Res Hum Retroviruses 2008;24(3):355-362.

17. Price MA, Wallis CL, Lakhi S, et al.: Transmitted HIV type 1 drug resistance among individuals with recent HIV infection in East and Southern Africa. AIDS Res Hum Retroviruses 2011;27(1):5-12.

18. Frentz D, van de Vijver D, Abecasis A, et al.: Patterns of transmitted HIV drug resistance in Europe vary by risk group. PLoS One 2014;9(4):e94495.

19. Sayan M, Sargin F, Inan D, et al.: Transmitted antiretroviral drug resistance mutations in newly diagnosed HIV-1 positive patients in Turkey. J Int AIDS Soc 2014;17(4 Suppl. 3): 19750.

20. SPREAD Programme: Transmission of drug-resistant HIV-1 in Europe remains limited to single classes. AIDS 2008;22: $625-635$.
21. Garcia-Lerma JG: Diversity of thymidine analogue resistance genotypes among newly diagnosed HIV-1-infected persons. J Antimicrob Chemother 2005;56:265-269.

22. Weinstock H, Zaidi I, Heneine W, et al.: The epidemiology of antiretroviral drug resistance among drug-naive HIV-1 infected persons in 10 U.S. cities. J Infect Dis 2004;189: 2174-2180.

23. Violin M, Cozzi-Lepri A, Velleca R, et al.: Risk of failure in patients with $215 \mathrm{HIV}-1$ revertants starting their first thymidine analog-containing highly active antiretroviral therapy. AIDS 2004;18:227-235.

24. Pingen M, Nijhuis M, Mudrikova T, et al.: Infection with the frequently transmitted HIV-1 M41L variant has no influence on selection of tenofovir resistance. J Antimicrob Chemother 2015;70(2):573-580.

25. Pingen M, Nijhuis M, de Bruijn JA, et al.: Evolutionary pathways of transmitted drug-resistant HIV-1. J Antimicrob Chemother 2011;66:1467-1480.

26. Shafer RW, Rhee SY, Pillay D, et al.: HIV-1 protease and reverse transcriptase mutations for drug resistance surveillance. AIDS 2007;21:215-223.

27. UNHCR official web page; www.unhcr.org/pages/49e48efa7f .html.

28. Inan D and Sayan M: Molecular epidemiology of HIV-1 strains in Antalya, Turkey. J Int AIDS Soc 2014;2;17(4 Suppl. 3): 19684.

29. Trafficking in human beings report of European Commission. https://ec.europa.eu/anti-trafficking/sites/antitrafficking/files/ trafficking_in_human_beings_-_eurostat_-_2014_edition.pdf.

Address correspondence to:
Murat Sayan
Clinical Laboratory
PCR Unit
University Hospital of Kocaeli
Eski Istanbul Yolu
Umuttepe Campus
41380 Izmit
Kocaeli
Turkey

E-mail: sayanmurat@hotmail.com 